\title{
Relato de caso de paciente com tetraplegia incompleta e seu processo de reabilitação
}

Case report of incomplete tetraplegy patient and its rehabilitation process

Caso clínico del paciente de tetraplegia incompleto y su proceso de rehabilitación

Raulcilaine Érica dos SANTOS ${ }^{1}$

Gustavo Faleiro BARBOSA ${ }^{1}$

Amanda Oliva SPAZIANI ${ }^{1}$

Flávio Henrique Nuevo Benez dos SANTOS ${ }^{2}$ Raissa Silva FROTA ${ }^{3}$

Luciana Lara Vicente PARREIRA ${ }^{1}$ Bianca Pereira de BRITO ${ }^{1}$

Victor Nagib Queiroz BALECH ${ }^{1}$

Fabielli Mioto LARROSSA ${ }^{1}$

André Victor Vian GARCIA ${ }^{1}$

Lara Maria Souza Marcondes COLOGNESI ${ }^{1}$

Maurício Caramel da SILVA ${ }^{1}$

Rullya Marson de Melo OLIVEIRA ${ }^{1}$

Victor Borges da SILVA ${ }^{1}$

Talita Costa BARBOSA 1

Hayani Yuri Ferreira Outi SANTOS

${ }^{1}$ Curso de Graduação em Medicina, Universidade Brasil - UB, 15600-000 Fernandópolis - SP, Brasil

${ }^{2}$ Docente do Curso de Medicina, Universidade Brasil UB 15600-000, Fernandópolis, São Paulo, Brasil ; Gerente Médico, Rede de Reabilitação Lucy Montoro, 15600-000 Fernandópolis - SP, Brasil

${ }^{3}$ Curso de Graduação em Medicina, Universidade de Rio Verde - UniRV, 75901-970 Goianésia - GO, Brasil

\section{Resumo}

Introdução: A tetraplegia é definida como uma paralisia que atinge simultaneamente os quatro membros. Já a lesão medular pode acometer multifatorialmente o paciente, o qual necessita de um conjunto de ações que vai desde o atendimento primário até a reabilitação. O objetivo deste trabalho é relatar um caso de lesão medular por efeito chicote com tetraplegia incompleta e o seu processo de reabilitação. Relato de caso: realizado por meio de coleta de dados por prontuário médico e levantamento de dados; sexo masculino, 23 anos. A tetraplegia incompleta entre C3 e C4 ocorreu devido a acidente em outubro de 2016 em uma lesão em chicote sem perda da consciência. Foi realizada uma artrodese para estabilização, quatro dias após, teve alta hospitalar. Iniciou a reabilitação e até dezembro de 2016 recuperou os movimentos grossos manipulativos das mãos, ainda sem sensibilidade. Em janeiro de 2017 começou a utilizar órtese de posicionamento de dedos e punho, realizou exercício para coordenação motora fina, aumento da amplitude de movimento e força. Em maio apresentava dominância manual direita, função manual preservada com dificuldade nas preensões, força muscular diminuída, alterações de sensibilidade, bom controle cervical e foram realizadas atividades com o objetivo de ortostatismo. Atualmente, o paciente encontra-se melhorando a habilidade já adquirida de ortostatismo. Conclusão: Como a lesão medular traz consigo muitas repercussões na vida do paciente espera-se que o cuidado seja integral, tendo como resultado a manutenção da sua saúde física e mental, proporcionando o desenvolvimento da autonomia para exercer plenamente suas atividades da vida diária.

Descritores: Quadriplegia; Reabilitação; Medula Espinal.

\section{Abstract}

Introduction: Quadriplegia is defined as a paralysis that simultaneously affects all four limbs. Spinal cord injury can multifactorially affect the patient, which requires a set of actions ranging from primary care to rehabilitation. The objective of this paper is to report a case of whiplash injury with incomplete quadriplegia and its rehabilitation process. Case Report: Performed through data collection by medical records and data collection; male, 23 years old. Incomplete quadriplegia between C3 and C4 occurred due to an accident in October 2016 in a whiplash injury without loss of consciousness. Arthrodesis for stabilization was performed four days later and was discharged from hospital. He began rehabilitation and until December 2016 recovered the manipulative movements of the hands, still without sensitivity. In January 2017, he started to use finger and wrist positioning orthosis, performed exercise for fine motor coordination, increased range of motion and strength. In May, he had right hand dominance, hand function preserved with difficulty in grasping, decreased muscle strength, sensitivity changes, good cervical control, and activities were performed with the aim of standing. Currently, the patient is improving the already acquired ability of orthostatism. Conclusion: As spinal cord injury has many repercussions on the patient's life, care is expected to be comprehensive, resulting in the maintenance of their physical and mental health, providing the development of autonomy to fully perform their activities of daily living.

Descriptors: Quadriplegia; Rehabilitation; Spinal Cord.

\section{Resumen}

Introducción: La cuadriplejia se define como una parálisis que afecta simultáneamente a las cuatro extremidades. La lesión de la médula espinal puede afectar multifactorialmente al paciente, lo que requiere un conjunto de acciones que van desde la atención primaria hasta la rehabilitación. El objetivo de este trabajo es informar un caso de lesión por latigazo cervical con cuadriplejia incompleta y su proceso de rehabilitación. Informe de Caso: realizado a través de la recopilación de datos por registros médicos y la recopilación de datos; masculino, 23 años. La cuadriplejia incompleta entre C3 y C4 se produjo debido a un accidente en octubre de 2016 en una lesión por latigazo cervical sin pérdida de conocimiento. La artrodesis para la estabilización se realizó cuatro días después y fue dada de alta del hospital. Comenzó la rehabilitación y hasta diciembre de 2016 recuperó los movimientos manipuladores de las manos, aún sin sensibilidad. En enero de 2017, comenzó a usar la ortesis de posicionamiento de los dedos y las muñecas, realizó ejercicios para la coordinación motora fina, aumentó el rango de movimiento y la fuerza. En mayo, tenía dominio de la mano derecha, la función de la mano se preservaba con dificultad para agarrar, la disminución de la fuerza muscular, los cambios de sensibilidad, el buen control cervical y las actividades se realizaban con el objetivo de estar de pie. Actualmente, el paciente está mejorando la capacidad ya adquirida del ortostatismo. Conclusión: Como la lesión de la médula espinal tiene muchas repercusiones en la vida del paciente, se espera que la atención sea integral, lo que resulta en el mantenimiento de su salud física y mental, proporcionando el desarrollo de autonomía para realizar plenamente sus actividades de la vida diaria.

Descriptores: Cuadriplejía; Rehabilitación; Médula Espinal.

INTRODUÇÃO

A tetraplegia é definida como uma paralisia que atinge simultaneamente os quatro membros. São sistematicamente consequências irreversíveis de patologias neurológicas ou neurocirúrgicas graves. Já a lesão medular pode acometer multifatorialmente o paciente, 0 
qual necessita de um conjunto de providências que vão que vai desde o atendimento primário até a reabilitação e reintegração do paciente na sociedade ${ }^{1}$.

Historicamente, a lesão medular está associada a preocupantes taxas de incidência e prevalência. Números recentes divulgados nos Estados Unidos mostram que aproximadamente 11.000 novos casos são registrados a cada ano, acrescenta-se, ainda, que outra informação divulgada informa que país tem um contingente de até 288.000 pessoas vivendo com lesão medular espinal. Tal situação, evidencia a frequência dos acidentes, com a consequente sequelas, acarretando ônus ao paciente e porquê não evidenciar o ônus ao sistema de saúde, principalmente quando se trata das lesões causadoras de tetraplegias completas. Quando se considera os casos acontecidos no Brasil, tem-se a lamentar a falta de dados como esses, pois os registros ainda são desconhecidos, em função da ausência de um sistema eficaz de notificação e registro, mesmo ao se considerar as altas taxas de acidentes automobilísticos, que constituem a principal causa de lesão medular ${ }^{2,3}$.

Estima-se que no Brasil a incidência de trauma raquimedular seja de 40 casos novos/ano/milhão de habitantes, extrapolando o cálculo, obtém-se a existência de cerca de 6 a 8 mil casos novos por ano. Esses números evidenciam que, desse total de vítimas, $80 \%$ são do sexo masculino, sendo que $60 \%$ se encontram entre os 10 e 30 anos de idade. $O$ trauma é a causa predominante, sendo responsável no país por mais de 10 mil novos casos de lesão medular, sendo que esse tipo de patologia de elevado impacto socioeconômico ${ }^{4,5}$.

A lesão medular é classificada a partir de uma padronização internacional determinada pela American Spinal Injury Association. Seu diagnóstico é baseado na avaliação da sensibilidade e da função motora, outra possibilidade é a classificação do paciente quanto ao tipo de lesão que pode ser completa ou incompleta, além de determinar o nível neurológico, e gerar um escore baseado nos achados sensitivos e motores ${ }^{5,6}$.

Esse tipo de lesão traz consigo determinados pontos de avaliação com relevância, destacando-se, entre eles: dor neuropática, alterações musculoesqueléticas, alterações vasculares, bexiga neurogênica, intestino neurogênico, ulceras por pressão e espasticidade e/ou automatismos ${ }^{7}$. A reabilitação para esse tipo de caso perpassa por uma série de eventos, manejos e profissionais.
Torna-se imprescindível o acompanhamento fisioterapêutico neurofuncional e respiratório, terapia ocupacional, órteses, adaptações para atividades básicas, cadeira de rosas e orientações para o paciente, família e cuidadores sobre as atividades da vida diária ${ }^{7,8}$.

O objetivo deste trabalho, portanto, é relatar um caso de lesão medular por efeito chicote com tetraplegia classificada como incompleta e o seu processo de reabilitação.

CASO CLÍNICO

Característica do paciente: sexo masculino e 23 anos de idade. Identificação da anomalia: tetraplegia incompleta entre C3 e C4, ocorrência do fato gerador da tetraplegia: acidente de trabalho ocorrido em outubro de 2016. Conforme relato do paciente, no ambiente de trabalho, ao passar por baixo da grade do ar condicionado colidiu com a fronte no exaustor do aparelho, caindo para trás, caracterizando, dessa forma, uma lesão em chicote, acidente esse que provocou a lesão na coluna. Nesse acidente, não houve perda da consciência, apenas dos movimentos e da sensibilidade. Imediatamente o socorro foi chamado e o acidentado foi levado para o hospital. Foi realizada uma artrodese por via anterior com intuito da estabilização das vértebras C3 e C4. Transcorrido quatro dias após a intervenção cirúrgica, o paciente foi encaminhado para casa. De outubro a dezembro de 2016, realizou trabalho de reabilitação e conseguiu recuperar os movimentos grossos das habilidades manipulativas das mãos, ainda sem sensibilidade e com sensação de calor intenso no corpo. A partir de janeiro de 2017, iniciou a reabilitação em um centro especializado. Para a reabilitação foi utilizado órtese de posicionamento de dedos e punho, praticou exercícios para coordenação motora fina, aumento da amplitude de movimento e força, fez, ainda, terapia robótica para melhorar aspectos motores, posturais e de movimento. Em maio de 2017, apresentava dominância manual direita, função manual preservada com dificuldade nas preensões, força muscular diminuída, alterações de sensibilidade, não possuía dor ou tremulação em corpo, bom controle cervical, controle de tronco regular e ausência de escoliose. Transcorridos seis meses, em agosto de 2017, foram realizadas atividades com o objetivo de colocar o paciente em ortostatismo, fortalecer tronco e membros, ficar em pé no andador e em prancha ortostática, realizando marcha em barra.

DISCUSSÃO

Cuidados especiais devem ser prática 
usual em pessoas com suspeita de trauma raquimedular, essas preocupações e tratamento deve ser iniciado imediatamente, ainda em ambiente pré-hospitalar, devendo seguir 0 protocolo do ABCDE, conforme transcrito a seguir: $A$ (abertura da via aérea e controle da coluna cervical); B (ventilação); C (circulação); D (disfunção neurológica); e E (exposição e ambiente). A avaliação da biomecânica serve como base para prever as lesões que sucederam na coluna cervical. A conduta de colocar o colar cervical deve, ainda ser precedida da medida do tamanho do colar. A proteção da coluna cervical de todos os pacientes traumatizados, até que seja eliminada a hipótese de qualquer lesão, mesmo que necessite manusear a via aérea, deve ser preocupação de qualquer socorrista ${ }^{1,9}$.

Além do desafiador impacto psíquico, social e econômico, a lesão medular espinal é caracterizada como uma complexa disfunção clínica e funcional, desencadeada pelo dano ao tecido nervoso medular, frequentemente por mecanismos traumáticos, como fratura-luxação de corpos vertebrais. Estes mecanismos invariavelmente repercutem com ampla e heterogênea alteração fisiológica, em que se destacam os prejuízos à função neuromotora como a incapacidade de locomoção em detrimento da paralisia completa de membros, além de ineficaz força muscular respiratória para ventilação voluntária. De acordo com dados do National Spinal Cord Injury Statistical Center, as mudanças nos últimos 40 anos dos paradigmas de assistência e reabilitação favoreceram a taxa de sobrevivência e de expectativa de vida das pessoas com lesão medular ${ }^{3,5}$.

Contudo, como a lesão medular acarretaproblemas ao paciente, modificando seus hábitos e modos de agir, espera-se que 0 cuidado seja absoluto, tendo como resultado a manutenção da sua saúde física e mental, propiciando o desenvolvimento da autonomia para exercer plenamente suas atividades da vida diária ${ }^{3,10}$.

Torna-se necessário a prática de atividades nos especializados e de alta complexidade, mas mostra-se, da mesma forma importante a atenção básica nesse tipo de anomalias, já que possui a tentativa de proporcionar um cuidado continuado com a integração dentro das redes de diferentes complexidades ${ }^{9}$.

Estudos aconselham que os procedimentos de reabilitação tenham seu início imediato assim que haja a estabilização da lesão medular e quanto mais rápido for 0 atendimento, melhor o impacto do processo de reabilitação. Isso posto, 0 a intervenção imediata no processo de reabilitação deve ter seu início desde a fase aguda, tão logo se estabilizem as condições clínicas, ainda nas unidades de terapia intensiva, a fim de minimizar complicações que podem ser danosa e custosas ${ }^{7,11}$.

\begin{tabular}{|c|c|}
\hline Destaca-se & entre \\
\hline $\begin{array}{l}\text { complicações do } \\
\text { destacam-se } \\
\text { cardiocirculatórias, }\end{array}$ & $\begin{array}{l}\text { trauma } \\
\text { as } \\
\text { hipotensão }\end{array}$ \\
\hline
\end{tabular}
disreflexia autonômica, disfunção urinária (bexiga neurogênica), gastrointestinais (intestino neurogênico) e úlceras por pressão. Os danos funcionais necessitam serem abordados na prevenção das tais complicações e incapacidades secundárias ${ }^{5,11}$.

Traça-se como fato relevante, ainda, que os profissionais multiprofissionais elaborem as metas terapêuticas e probabilidade na recuperação funcional em função dos níveis de lesão, mesmo porque o tratamento terapêutico é função dos órgãos lesionados. Torna-se importante, também, orientar tarefas, estimulando o treino básico de atividades em cadeira de rodas, ou práticas que facilitem a vida cotidiana do paciente acometido por este tipo de lesão $0^{7,8,12}$.

Também se torna importante explicar ao como aplicar os princípios de conservação de energia, além de outros conceitos como a necessidade de utilizar proteção articular e quais vantagens da aplicação da biomecânica durante as mudanças posturais, as transferências e atividades que demandem maior força e sobrecarga, principalmente a articular? .

O tratamento cirúrgico deve considerar a estabilização completa do paciente, com a finalidade de reduzir as fraturas e descomprimir a medula ${ }^{9}$.

\section{REFERÊNCIAS}

1. Silva EM, Tobias FLM, Rufine M, Zardo KCB. Avaliação da qualidade de vida de jogadores de rugby com lesão medular traumática do Distrito Federal: estudo piloto. Rev Ciênc Saúde. 2019;1(1):8-12.

2. Campos MF, Ribeiro AT, Listik S, Pereira CAB, Andrade Sobrinho J, Rapoport A. Epidemiologia do traumatismo da coluna vertebral. Rev Col Bras Cir. 2008;35(2):88-93.

3. Macedo FS, Paz CCSC, Rocha AF, Miosso CJ, Carvalho HB, Mateus SRM. Novas perspectivas de fisioterapia respiratória em lesão medular: uma revisão sistemática. Acta Paul Enferm. 2017;30(5):554-64.

4. Stripling TE. The cost economic consequences 
of traumatic spinal cord injury. Paraplegia News. 1990;8:50-4.

5. Soligo C, Sebben AA. Lesão medular traumática: mudanças biopsicossociais e suas consequências. Unoesc Ciênc. ACBS. 2019;10(1):67-74.

6. Barros Filho TEP. Avaliação padronizada nos traumatismos raquimedulares. Rev Bras Ortop. 1994;9(3):99-106.

7. Ferreira TCPGA, Rodrigues RM, Cruz PM, Ribeiro MFM, Prudente COM. Relação entre qualidade de vida e capacidade funcional de pacientes com lesão medular após 6 meses de reabilitação. Anais do $5^{\circ}$ Congresso de Ensino, Pesquisa e Extensão da Universidade Estadual de Goiás; 2015, out. 20-22; Pirenópolis. Anápolis: UEG; 2015.

8. Ettinger Larissa Feitosa Cirino et al. Qualidade de vida das vítimas de trauma raquimedular atendidas em centros de reabilitação de Aracaju. Interfaces Cient Saúde Ambiente. 2017;5(2):53-62.

9. Correia CA, Abreu RNDC, Oliveira M, Moreira VT, Rodrigues AMU. Caracterização dos pacientes em uso de colar cervical. Rev Enferm UFPI. 2016;5(2):28-31.

10. Melo-Neto JS, Vidotto LEL, Gomes FC, Morais DF, Tognola WA. Caracterização e aspectos clínicos de pacientes com traumatismo raquimedular submetidos a cirurgia. Rev Bras Ortop. 2017;52(4):479-90.

11. Campoy LT, Rabeh SAN, Nogueira PC, Vianna PC, Miyazaki MY. Práticas de autocuidado para funcionamento intestinal em um grupo de pacientes com trauma raquimedular. Acta Fisiátr. 2016;19(4):228-32.

12. Alves CL, Faria RLBC, Vulcano IA, Bastos AMTN, Silva ÁMS. Trauma raquimedular em paciente de 12 anos com compressão de canal medular sem déficits neurológicos. Rev Patol Tocantins. 2018;5(1):41-5.

\section{CONFLITO DE INTERESSES}

Os autores declaram não haver conflitos de interesse

\section{AUTOR PARA CORRESPONDÊNCIA}

\section{Amanda Oliva Spaziani}

E-mail: spazianimedicina@gmail.com

Submetido em 11/09/2019

Aceito em 20/08/2020 